\title{
LETTER \\ Robust Hybrid Finger Pattern Identification Using Intersection Enhanced Gabor Based Direction Coding
}

\author{
Wenming YANG $^{\dagger \mathrm{a})}$, Wenyang $\mathrm{JI}^{\dagger \mathrm{b})}$, Nonmembers, Fei $\mathrm{ZHOU}^{\dagger c}$, Member, and Qingmin LIAO $^{\dagger \mathrm{d})}$, Nonmember
}

SUMMARY Automated biometrics identification using finger vein images has increasingly generated interest among researchers with emerging applications in human biometrics. The traditional feature-level fusion strategy is limited and expensive. To solve the problem, this paper investigates the possible use of infrared hybrid finger patterns on the back side of a finger, which includes both the information of finger vein and finger dorsal textures in original image, and a database using the proposed hybrid pattern is established. Accordingly, an Intersection enhanced Gabor based Direction Coding (IGDC) method is proposed. The Experiment achieves a recognition ratio of $98.4127 \%$ and an equal error rate of 0.00819 on our newly established database, which is fairly competitive.

key words: finger vein identification, multi-feature infrared finger pattern, vascular biometrics, finger dorsal textures

\section{Introduction}

Automated identification of humans using finger patterns has been increasingly investigated because of the tremendous growth in various security applications. Naoto Miura and Akio Nagasaka proposed two classical methods of person identification based on finger-vein patterns, i.e. repeated line tracking [1] and maximum curvature [2]. Besides, multi-modal traits have also been explored. Yang and Huang [3] presented a multi-modal personal identification system using finger vein and finger dorsal images with feature-level fusion. The traditional feature-level fusion strategy is limited and expensive, which often needs two or more cameras to acquire finger vein and finger dorsal textures. To solve the problems, we find that more texture information can be acquired when fingers are viewed from the back side, as Fig. 1.b shows. Thus we propose a compromise method to achieve both finger vein and finger dorsal texture features simultaneously using only one camera, which is comparatively cheap while preserving texture information as much as possible.

Key contributions of this paper can be summarized as in the following:

a This paper investigates the possibility of using image that

Manuscript received April 4, 2016.

Manuscript revised June 14, 2016.

Manuscript publicized July 6, 2016.

${ }^{\dagger}$ The authors are with the Shenzhen Key Lab. of Information Sci\&Tech/Shenzhen Engineering Lab. of IS\&DCP Department of Electronic Engineering/Graduate School at Shenzhen, Tsinghua University, China.

a) E-mail: yangelwm@163.com

b) E-mail: jiwenyang666@163.com

c) E-mail: flying.zhou@163.com

d)E-mail: liaoqm@tsinghua.edu.cn

DOI: $10.1587 /$ transinf.2016EDL8074

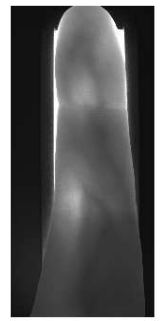

a

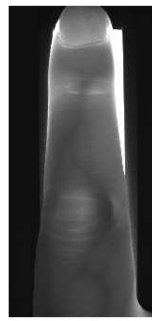

b
Fig. 1 (a) is the traditional inside near-infrared view of finger vein, (b) shows our proposed hybrid pattern

contains hybrid finger patterns viewed from the back side of fingers, which provides a new perspective in finger pattern identification.

b This paper provides the first publicly available database with hybrid patterns captured from the back side of the finger, from 126 different subjects. We hope that it helps to advance further research in this area.

c According to the new established database, an Intersection enhanced Gabor based Direction Coding (IGDC) method is proposed, which takes into account the magnitude result of gabor filtering.

The rest of this paper is organized as follows. Section 2 describes our Image acquisition device and the ROI Extraction method briefly. Section 3 shows some classical algorithms in textural feature extraction in finger vein images, such as gabor competitive coding, local binary patterns, local maximum curvature, etc. Section 4 details our proposed IGDC method in detail. Section 5 shows our experiments results using the algorithms described in Sects. 3 and 4 on the database proposed in this paper. Finally, the key conclusions from this paper are summarized in Sect. 6.

\section{Image Acquisition and Region of Interest Extraction}

Figure 2.a shows our finger vein and finger dorsal texture imaging device which consists of a black JAI camera based on CCD image sensor and one rectangular near-infrared illuminant. As Fig. 3 shows, the principle is that the nearinfrared light penetrates the finger and those passing through the veins will be absorbed by the hemoglobin in the blood to some extent, so the part of images where the vein is will be darker than other places. The pressed texture region is 


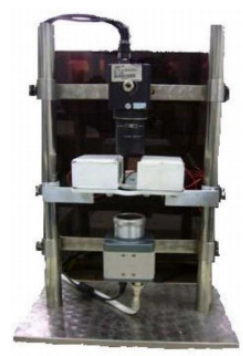

a

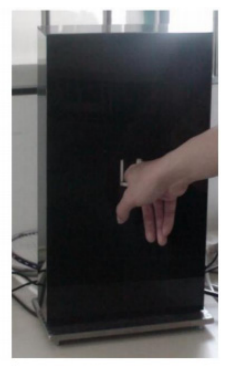

b
Fig. 2 (a) is our designed image acquisition device, (b) shows the image acquisition process

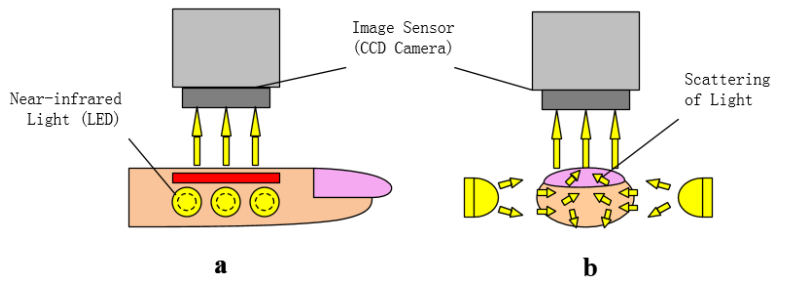

Fig. 3 (a) and (b) are the block diagrams showing the side view and front view of our imaging principle respectively

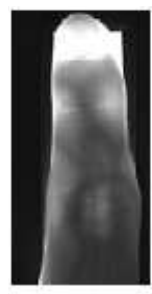

a

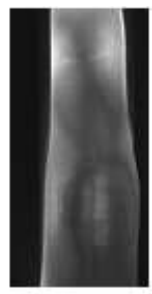

b

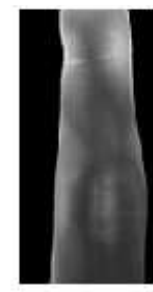

c

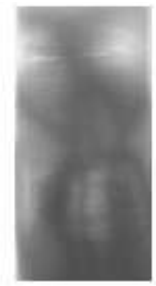

d
Fig. 4 (a) is the original image, (b) and (c) are the intermediate process, (d) is the final extracted region

thinner compared with the bulged region, with less scattering this part looks brighter. Figure 2.b also shows the image acquisition process.

Since further experiments are done on the preprocessed images, region of interest extraction plays a really important part in the whole identification procedure. This procedure consists of 3 steps concretely as the following. As Fig. 4 shows, Fig. 4.a shows the acquired original image, Fig. 4.b and Fig. 4.c show the intermediate process, and Fig.4.d shows the final extracted region. Refer to [10] for more concrete explanations.

\section{Feature Extraction and Matching}

\subsection{Local Binary Patterns}

As a classical texture extraction method, local binary pattern [10] is utilized as a comparison to test the reliability of the database. Given a pixel in the image, LBP codes are computed by comparing it with its neighbors.

$$
L B P_{P, R}=\sum_{p=0}^{P-1} s\left(g_{p}-g_{c}\right) 2^{p}, s(x)=\left\{\begin{array}{l}
1, x \geq 0 \\
0, x<0
\end{array}\right.
$$

In the above equation, $g_{c}$ is the value of central pixel, $g_{p}$ is the value of its neighbors, $\mathrm{P}$ is the total number of involved neighbors, and $\mathrm{R}$ is the radius of the neighborhood. After the coding process, a pixel by pixel compare is applied to compute the matching rate between two pictures.

\subsection{Local Maximum Curvature}

The experiments done in Sect. 5 also use the local maximum curvature algorithm [2] to extract features from images as a comparison. As a famous method in finger vein identification, it can extract the centerlines of the veins consistently without being affected by the fluctuations in vein width and brightness, so its pattern matching is highly accurate. Three steps are included in the algorithm: extraction of the center positions of veins, connection of the center positions and labeling of the image. The curvature calculation can be represented as follows.

$$
\kappa(z)=\frac{d^{2} P_{f}(z) / d z^{2}}{\left\{1+\left(d P_{f}(z) / d z\right)^{2}\right\}^{\frac{3}{2}}}
$$

$P_{f}(z)$ is defined as a cross-sectional profile acquired from the original image at any direction and position, where $\mathrm{z}$ is a position in a profile.

In the matching step, scores indicating the probability that the center positions on veins are assigned to each center position. A score, $S_{c r}\left(z_{i}^{\prime}\right)$, is defined as follows:

$$
S_{c r}\left(z_{i}^{\prime}\right)=\kappa\left(z_{i}^{\prime}\right) \times W_{r}(i)
$$

Where $W_{r}(i)$ is the width of the region where the curvature is positive and one of the $z_{i}^{\prime}$ is located.

\subsection{Gabor Competitive Coding}

The experiments in this paper use the $2 \mathrm{D}$ gabor filter based feature extraction method to exploit local direction information from the simultaneously acquired finger vein and finger dorsal images. Gabor filter is defined as follows.

$$
\begin{aligned}
& G\left(x, y, \gamma, \sigma, f_{0}, \theta\right) \\
& \quad=\frac{\gamma}{2 \pi \sigma^{2}} \exp \left\{-\frac{1}{2}\left(\frac{x_{\theta}^{2}+\gamma^{2} y_{\theta}^{2}}{\sigma^{2}}\right)\right\} \exp \left(j 2 \pi f_{0} x_{\theta}\right)
\end{aligned}
$$

Six directions are selected, and thus six filters are convoluted with the original picture. For each pixel, the orientation of the filter whose convolution result is minimum is used as the feature code. This kind of feature codes are completely independent of the contrast and the brightness of the capture devices. With the competitive rule, the algorithm is actually more robust to different capturing environments and devices. For more details refer to [11].

To match two coded pictures, the feature codes are transformed to 3-bit binary codes, and bitwise matching 
method is used to compute the similarity of the two pictures. A score is given representing this resemblance.

\section{Intersection Enhanced Gabor Based Direction Cod- ing}

Gabor filters have been widely used as an effective tool for feature extraction in many biometric systems. The experiments in this paper use the 2D gabor filter based feature extraction method to exploit local direction information from the simultaneously acquired finger vein and finger dorsal images. Empirically, six directions are selected, and thus six filters are convoluted with the original image respectively. For the dark region on the path of finger vein, the value resulted from the process of convolution with the corresponding direction gabor filter is usually the smallest. Therefore, traditional gabor based direction coding method adopt this direction as the feature code. Finally, the feature map is composed of directions labeled from 1 to 6 .

However, this method doesn't take full advantage of the bright horizontal information of the finger dorsal texture. The original gabor competitive coding method only takes into account the vertical orientation of finger veins, and ignores the horizontal bright finger dorsal textures. Here we use the magnitude of gabor filtering and try to extract the intersection points where the horizontal bright lines and vertical dark lines intersect.

The proposed method is based on the gabor convolution results that the intersection points of the dark vertical finger vein and the bright horizontal finger dorsal textures will result in two extreme values after the above six direction convolution processes, one very small in the direction of the finger vein and the other fairly large in the direction of the finger dorsal texture. And the intersection points can be detected by sensing how large the difference between the two values is. Firstly, a threshold is determined by the magnitude of the six filtering results as follows.

$$
T=\alpha \cdot\left(\max _{i=1,2, \ldots, 6} \operatorname{Mag}\left(I * G_{i}\right)-\min _{i=1,2, \ldots, 6} \operatorname{Mag}\left(I * G_{i}\right)\right)(5)
$$

$I$ represents the original image. $G_{i}$ represents the ith direction gabor filter and the symbol. $*$ represents the convolution operation. The parameter $\alpha$ is empirically set as 0.4 . And in our experiments, the threshold of $\mathrm{T}$ is $[0,44]$. For each pixel, the difference between the maximum and the minimum value is calculated and then it is compared with the above threshold. The direction of pixels that has the difference value larger than the threshold are identified as intersection points and labeled as 7 . Then the final feature map is composed of the numbers coded from 1 to 7 . As Fig. 5 shows, the regions in the red square contain many intersection points. Three examples of intersection points are given with their two main directions labeled by the red arrows. Figure 5.b show all the intersection points extracted by our method.

Furthermore, By attaching these intersection points a higher weight in matching, we can make the most of the

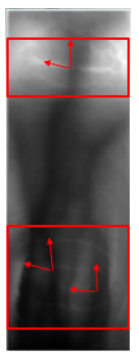

a

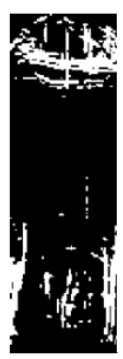

b.
Fig. 5 (a) The intersection points which have two orientations and (b) the intersection points extracted from the original image

information the image includes. The matching process can be represented as follows.

$S(R, T)=\max _{\forall i \in[-w, w], \forall j \in[-h, h]}\left(\frac{\sum_{x=1}^{m} \sum_{y=1}^{n} \odot(R(x+i, y+j), T(x, y))}{2 *(m+n)}\right)$

The function $\odot(J, K)$ is defined as follows.

$$
\odot(J, K)=\left\{\begin{array}{l}
0(J \neq K) \\
1(J=K \neq 7) \\
2(J=K=7)
\end{array}\right.
$$

The proposed matching step is an intersection enhanced algorithm, where we increase the weight of the matched intersection points to achieve more convictive result.

\section{Experimental Results}

In this section, we evaluate the proposed IGDC method on our newly established database. This database contains 126 hybrid finger vein and finger dorsal texture samples using the near-infrared technique. For comparison, another database only containing the finger vein pattern samples is established acquired from the other side of the finger. Region of interest of the images is normalized to $200 * 100$ pixels. The sample subjects are collected twice in an interval. 126 samples are chosen as the register samples and the other 126 are the testing set respectively. The experiment is conducted on Matlab2014a platform with $3.30 \mathrm{GHz}$ CPU. The recognition rate and the error equation rate are adopted here as the performance indicator of the hybrid vein pattern method.

As Fig. 6 shows, the feature map of Fig. 6.a is Fig. 6.b. 6 directions are selected and the intersection points are marked as 7. The bright white points in Fig. 6.b show the position of the intersection points. For comparison, other three classical texture feature extraction methods are tested both on the newly established database and the traditional database. Due to the fact that the IGDC method is proposed especially for the new database to add the information of horizontal bright finger dorsal textures, it is not tested on the traditional database [11]. Figure 7 shows the ROC curves of different algorithms. It can be seen that with the finger 


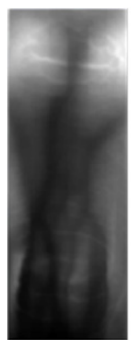

a

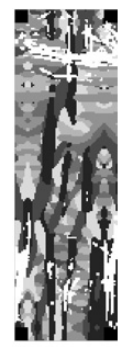

b
Fig. 6 (a) is the original input image; (b) is the corresponding IGDC feature, the brightest white points shows the position of the intersection points

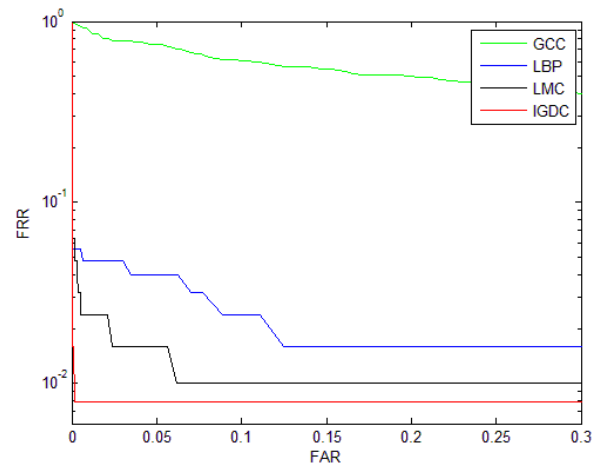

Fig. 7 The ROC curves of GCC, LBP, LMC and our proposed IGDC

Table 1 Performance of different algorithms

\begin{tabular}{|l|l|l|l|l|l|}
\hline & & LBP & LMC & GCC & IGDC \\
\hline Traditional & Accuracy(\%) & 90.48 & 93.65 & 91.27 & \\
\cline { 2 - 6 } Database & EER & 0.079 & 0.055 & 0.064 & \\
\hline Established & Accuracy(\%) & 96.83 & 97.62 & 95.24 & 98.41 \\
\cline { 2 - 6 } Database & EER & 0.032 & 0.022 & 0.039 & 0.008 \\
\hline
\end{tabular}

dorsal texture information added, the IGDC method shows more excellent performance in equal error rate (EER) on the new database than GCC [4], LBP [10] and LMC [2].

Furthermore, experiments on the two whole databases are conducted and the results of different algorithms are shown in Table 1. "Accuracy" in the table means the "recognition rate" of the algorithms respectively.

As is shown in Table 1, every algorithm performs better on the newly established database in the identification accuracy and EER. Since the two databases are acquired with the same device and in the same condition, they are comparable to each other. The result reveals that experiments conducted on the newly established database, with extra finger dorsal textures information, tends to show better performance. Besides, for the new database, the proposed algorithm appears to take the best advantage of the information in the image and achieves the highest identification accuracy and the lowest EER.

\section{Conclusion}

This paper has successfully investigated the possibility of employing hybrid finger pattern images for the biometric identification, which include both the feature of finger vein and finger dorsal textures captured from the back side of the finger. The experimental results illustrated in this paper, on the newly established database, can achieve promising results. Furthermore, an algorithm is accordingly proposed especially for the new database. This method takes better advantage of the information of finger vein and finger dorsal textures and achieve better performance than any other texture extraction algorithms in both identification accuracy and EER.

\section{Acknowledgements}

This work was supported in part by the Natural Science Foundation of China under Grant No.61471216 and in part by the Special Foundation for the Development of Strategic Emerging Industries of Shenzhen under Grant No.JCYJ20150831192224146 and No. JCYJ20150601165744635

\section{References}

[1] N. Miura, A. Nagasaka, and T. Miyatake, "Feature extraction of finger-vein patterns based on repeated line tracking and its application to personal identification," Machine Vision and Applications, vol.15, no.4, pp.194-203, 2004.

[2] N. Miura, A. Nagasaka, and T. Miyatake, "Extraction of finger-vein patterns using maximum curvature points in image profiles," IEICE Trans. Inf. \& Syst., vol.E90-D, no.8, pp.1185-1194, 2007.

[3] W. Yang, X. Huang, F. Zhou, and Q. Liao, "Comparative competitive coding for personal identification by using finger vein and finger dorsal texture fusion," Information Sciences, vol.268, pp.20-32, 2014.

[4] W.-K. Kong and D. Zhang, Competitive coding scheme for palmprint verification," Proc. 17th Inter national Conference on Pattern Recognition, 2004. ICPR 2004. IEEE, vol.1, pp.520-523, 2004.

[5] A. Kumar and Y. Zhou, "Human identification using finger images," IEEE Trans. Image Process., vol.21, no.4, pp.2228-2244, 2012.

[6] A. Kumar, "Importance of being unique from finger dorsal patterns: Exploring Minor Finger Knuckle Patterns in verifying Human Identities," vol.9, no.8, pp.1288-1298, 2014.

[7] W. Yang, C. Qin, and Q. Liao, "A Database with ROI Extraction for Studying Fusion of Finger Vein and Finger Dorsal Texture," Biometric Recognition, vol.8833, pp.266-270, Springer International Publishing, Cham, 2014.

[8] W. Yang, Q. Rao, and Q. Liao, "Personal identification for single sample using finger vein location and direction coding," 2011 International Conference on Hand-Based Biometrics, pp.1-6, 2011.

[9] W. Yang, W. Ji, and Q. Liao, "Significance of being unique from finger patterns: Exploring Hybrid Near-infrared Finger Vein and Finger Dorsal Patterns in Verifying Human Identities," Biometric Recognition, Lecture Notes in Computer Science, vol.9428, pp.529-535, Springer International Publishing, Cham, 2015.

[10] T. Ojala, M. Pietikainen, and T. Maenpaa, "Multiresolution GrayScale and Rotation Invariant Texture Classification with Local Binary Patterns," IEEE Trans. Pattern Anal. Mach. Intell., vol.24, no.7, pp.971-987, 2002.

[11] Tsinghua University Finger Vein and Finger Dorsal Texture Database1THU-FVFDT1, http://www.sz.tsinghua.edu.cn/labs/vip1/ thu-fvfdt.html 